\title{
Ultraschall-Tomografie zur Inline-Überwachung von Kunststoffschmelzen
}

\author{
Norbert Halmen, Christoph Kugler, Thomas Hochrein, Karsten Kretschmer, \\ Peter Heidemeyer, Martin Bastian \\ SKZ - Das Kunststoff-Zentrum, Friedrich-Bergius-Ring 22, 97076 Würzburg, Deutschland \\ (n.halmen@sk.de)
}

\section{Zusammenfassung}

Die prozessintegrierte Ermittlung von Prozess- und Produktkenngrößen ist eine große Hilfe bei der Bewertung oder Optimierung neuer Verfahren. Aus diesem Grund wurde ein UltraschallProzesstomografie-System entwickelt, welches die lokale Füllstoffverteilung von Kunststoffschmelzen bildgebend wiedergibt. Gegenstand der Untersuchung waren extrudierte Vollstäbe aus Polypropylen mit radialen Füllstoffgradienten. Während der Extrusion wurden Schallgeschwindigkeit und Dämpfung der Kunststoffschmelze ermittelt und über einen modifizierten Rekonstruktionsalgorithmus nach Radon als 2D-Schnittbilder aufbereitet. Trotz der Herausforderung einer hohen Dämpfung von $60 \mathrm{~mm}$ gefültter PP-Schmelze, ist eine akzeptable Bildgebung möglich. Ein wichtiger Faktor für die Bildqualität der tomografischen Rekonstruktion ist dabei der Öffnungswinkel der verwendeten Ultraschallwandler. Zusätzlich wurde eine Simulationsumgebung in Matlab entwickelt, welche als Testplattform für das Messsystem diente.

Keywords: Ultraschall, Tomografie, Bildrekonstruktion, Prozessüberwachung, Gradientenwerkstoffe

\section{Einleitung}

\section{Ultraschall zur Prozessüberwachung}

Bei der Prüfung mit Ultraschall (US, akustische Wellen im Frequenzbereich von $20 \mathrm{kHz}$ bis $1 \mathrm{GHz}$ ) macht man sich die frequenzabhängige Dämpfung aufgrund viskoser Verluste sowie Streu- und thermoelastische Effekte im Ausbreitungsmedium zunutze [1]. Nachdem US sich v. a. zu Diagnosezwecken im medizinischen Bereich durchgesetzt hat, ist er mittlerweile auch in der zerstörungsfreien Prüfung und der Prozess- und Qualitätsüberwachung im Kunststoffbereich angekommen [1]. Die Erzeugung von US-Signalen basiert dabei meistens auf dem piezoelektrischen Effekt [2, 3]. Da Messungen nahezu in Echtzeit möglich sind [4], eignet sich US u. a. als zerstörungsfreies Prüfverfahren für Inline-Messungen in Kunststoffschmelzen. In Flüssigkeiten und Kunststoffschmelzen breitet sich US hauptsächlich in Form von Longitudinalwellen aus. Um die Prüfung in heißen Medien zu ermöglichen, müssen die US-Prüfköpfe speziell konstruiert oder durch entsprechende Maßnahmen geschützt sein. Aufgrund der hohen Temperaturanforderungen werden oft Vorlaufstrecken eingesetzt, welche eine thermische Entkopplung zwischen den temperatursensitiven USWandlern mit ihren Klebeverbindungen und der heißen Schmelze erlauben. Die Vorlaufstrecken bestehen aus Materialien, welche eine gute Schallübertragung aber geringe Wärmeleitfähigkeit besitzen, wie etwa Glas, Keramik, spezielle Stahllegierungen oder Hochleistungskunststoffe [1, 2]. So können z. B. Polyimid (PI) oder Polyetheretherketon (PEEK) als Kopplungswerkstoffe bei Temperaturen bis $250^{\circ} \mathrm{C}$ eingesetzt werden. Zur Qualitätskontrolle an Polymerschmelzen kann die US-Messung in Transmission (Sender und Empfänger stehen sich gegenüber) eingesetzt werden. Der ausgesendete Puls wird beim Durchlaufen der Schmelze gedämpft und seine Laufzeit verändert. Hierdurch sind Rückschlüsse auf die viskosen und elastischen Eigenschaften der Kunststoffschmelze möglich. Insbesondere Signale mit Frequenzen zwischen $100 \mathrm{kHz}$ und $10 \mathrm{MHz}$ können sich auch in hochgefüllten Compounds ausbreiten [5]. In bisherigen Untersuchungen wurde u. a. gezeigt, dass sich die Volumenanteile von Füllstoffen in Compounds mit unterschiedlichen Basispolymeren anhand der Schallgeschwindigkeit ermitteln lassen [5, 6]. Diese ist außerdem abhängig von Druck und Temperatur. Ebenso hat die Streuung an Partikeln einen Einfluss auf die Dämpfung des US-Signals. Dadurch ist eine Aussage über die Füllstoffgrößenverteilung bzw. über Agglomeratbildung möglich [7]. 


\section{Polymere Gradientenwerkstoffe}

Bei der Extrusion von Halbzeugen aus Kunststoff werden die späteren Bauteileigenschaften durch Zugabe von Zuschlagstoffen gezielt modifziert, um etwa besondere Anforderungen an mechanische Eigenschaften, Licht- oder Chemikalienbeständigkeit zu erfüllen. Die funktionellen Additive sind dabei typischerweise homogen über das komplette Bauteil verteilt. Eine gezielte Verteilung der Zuschlagstoffe im Bauteil kann entweder mittels Co-Extrusion in Form scharf umgrenzter Schichtsysteme mit Konzentrationssprüngen an den Grenzflächen [8] oder durch diskontinuierliche Verfahren erreicht werden [9, 10, 11]. Ein kontinuierliches Verfahren zur Extrusion von Vollstäben aus Polypropylen (PP) mit radialer Gradierung von Füllstoffen befindet sich in der Entwicklung [12]. Da die optimale Einstellung des Gradienten (keine lokale Verschiebung, keine Agglomeration der Additive) maßgeblich über die Bauteileigenschaften des finalen Halbzeugs entscheidet bzw. eindeutige Rückschlüsse auf die Güte des Herstellungsprozesses zulässt, ist die kontinuierliche Überwachung des Extrusionsprozesses von Vorteil. Die typische Prüfung erfolgt nach der Herstellung im Labor. Dabei können die Gradienten optisch per Sichtprobe oder Mikroskop überprüft werden. Eine genauere Methode stellt der Einsatz eines Röntgen-Computertomografen (CT) dar. Allerdings liegt hier zwischen der Probennahme (inkl. Abkühlen des Vollstabs) und dem Vorliegen eines Messergebnisses eine sehr lange Totzeit im Bereich von Stunden vor, wodurch eine Prozessentwicklung und -optimierung sehr langwierig ist. Zudem lassen sich sehr kleine Dichteunterschiede, wie sie z. B. bei Polymerblends vorliegen, mit der CT nicht auflösen.

\section{Ultraschall-Prozesstomografie (US-PT)}

Für eine bildgebende Echtzeit-Prozesskontrolle wurde ein US-PT-System entwickelt. Dieses benötigt im Gegensatz zu einer Röntgen-CT keine Schutzvorrichtungen und kann direkt per Adapter in den Prozess integriert werden.

Vergleichbare Ansätze existieren bereits im medizinischen Bereich für den Einsatz zur Mammografie [13], im Kunststoffbereich, um etwa die Temperaturverteilung in einem Schmelzekanal aufzulösen [14], oder bei der zerstörungsfreien Bauteilprüfung [15]. Eine auf US-Tomografie basierende Umsetzung zur Bestimmung der Füllstoffverteilung in Kunststoffschmelzen war bisher nicht bekannt.

\section{Experimenteller Teil}

\section{Messsystem}

Das System wurde an die Geometrie der Vollstäbe angepasst und besteht aus einem Sensorring mit einem Innendurchmesser von $60 \mathrm{~mm}$, welcher den Schmelzekanal kurz vor dem Austritt der formgebenden Düse bildet. Aufgrund der hohen Signaldämpfung von gefüllten Kunststoffschmelzen ergeben bei einem Durchmesser von $60 \mathrm{~mm}$ höhere Anforderungen an die Sendeleistung eines US-Wandlers. Um im Schmelzekanal eine gute Bildgebung mittels Tomografie zu ermöglichen, muss jeder Ortspunkt aus möglichst vielen verschiedenen Richtungen durchschallt werden [16]. Dies gelingt entweder mit einer großen Anzahl an Wandlern und/oder mit einem breiten Abstrahlwinkel pro Wandler. Eine größere Wandleranzahl bei der vorgegebenen Geometrie wäre gleichbedeutend mit kleineren Aperturen und somit auch größeren Öffnungswinkeln. Allerdings sinkt mit der Wandlerapertur auch die einbringbare Schalleistung aufgrund der kleineren Kontaktfläche zur Schmelze.

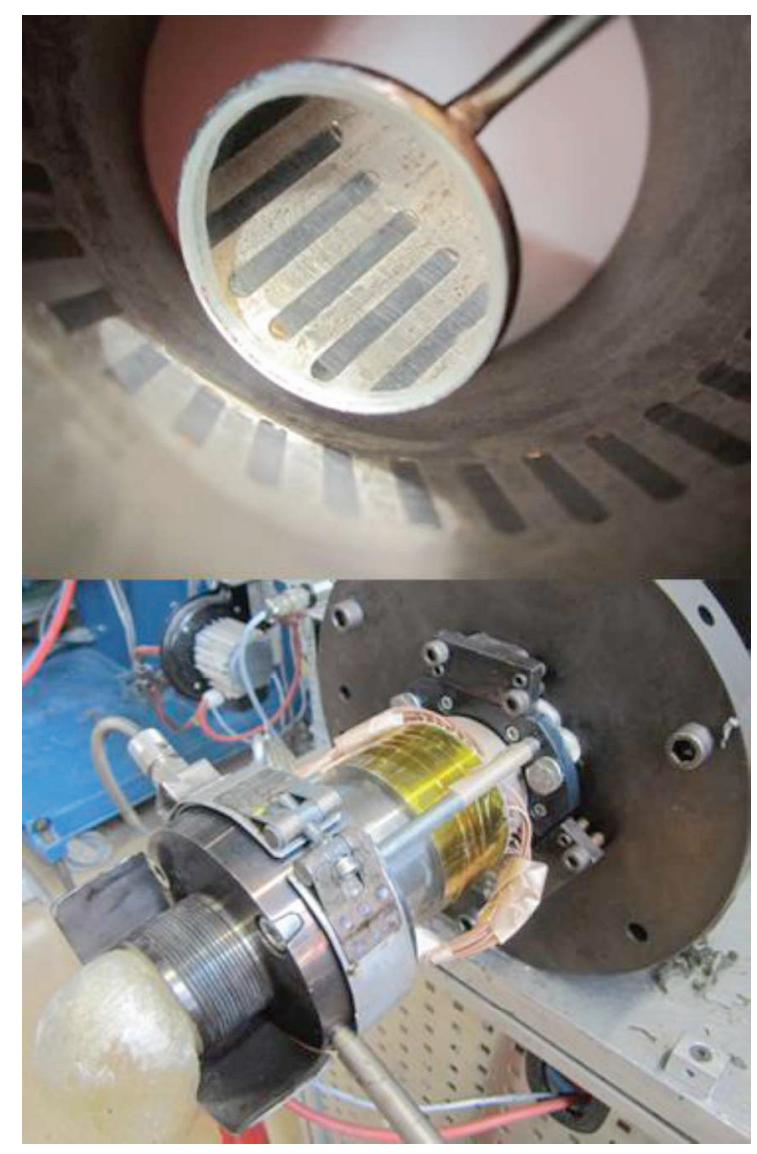

Abb. 1: US-Wandler des US-PT-Systems (oben) und am Gradientenwerkzeug montierter US-PT-Adapter (unten) 
Um einen guten Kompromiss zu bilden, wurde das US-PT-System mit 40 äquidistanten Wandlern mit Aperturen von $2 \mathrm{~mm} \times 14 \mathrm{~mm}$ (Breite $x$ Länge) ausgestattet, deren Vorlaufstrecken in direktem Schmelzekontakt stehen. Die schmale Breite senkrecht zur Fließrichtung soll dabei einen größeren Öffnungswinkel in der Schnittbildebene des Tomografen bewirken. Die größere Ausdehnung entlang der Fließrichtung der Schmelze bewirkt nur einen sehr kleinen Öffnungswinkel senkrecht zur Schnittbildebene, vergrößert aber die Kontaktfläche der Wandler und somit die einbringbare Schallleistung pro Wandler. Abb. 1 zeigt das Sensorsystems von innen sowie den an das Gradientenwerkzeug montierten Adapter.

Die US-Wandler werden mit einer RechteckBurst-Funktion bei einer Frequenz von $2,12 \mathrm{MHz}$ und mit einer Spannung zwischen 12 und $102 \mathrm{~V}$ angeregt. Die Anzahl der BurstWiederholungen ist dabei einstellbar. Simulationen des Herstellers ergeben eine um $6 \mathrm{~dB}-$ also etwa um die Hälfte - niedrigere Signalintensität bei einer Abweichung von $15^{\circ}$ zum Lot. Dieser Wert dient als Kenngröße für die Festlegung des Öffnungswinkels auf $30^{\circ}$. Der Adapter und die Wandler sind für die anspruchsvollen Prozessbedingungen bei der Herstellung der Gradientenwerkstoffe ausgelegt und arbeiten bei Temperaturen bis zu $250{ }^{\circ} \mathrm{C}$ und einem Druck bis 20 bar. Die Systemelektronik (Inoson PCM 12343, vgl. Abb. 2) ermöglicht neben der Ansteuerung der Wandler eine Aufnahme von Messsignalen mit einer Abtastrate von maximal $100 \mathrm{MHz}$ und bis zu 16384 Abtastpunkten.

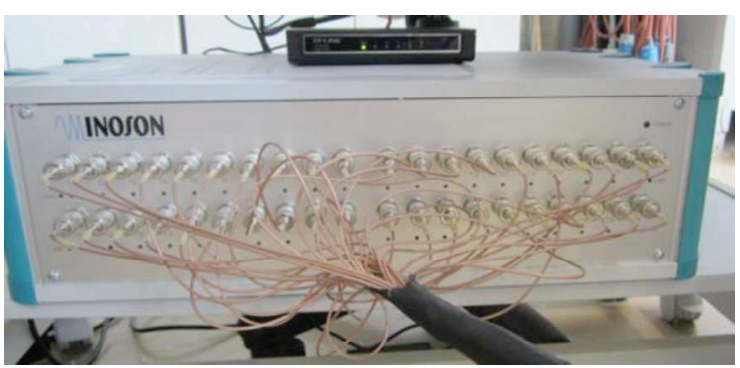

Abb. 2: Systemelektronik des US-PTSystems (Inoson PCM 12343)

Schnelle Funktionsprüfungen und die Optimierung einzelner Signalparameter wurden in der Herstellersoftware auf LabView-Basis durchgeführt. Die Steuerung des Messsystems bei tomografischen Messungen beginnend bei der Signalerfassung über die Auswertung bis hin zur Darstellung der Ergebnisse erfolgt über eine in der Programmiersprache Matlab entwickelte grafische Benutzeroberfläche. Die Messdauer für eine vollständige Messung mit allen Wandlern dauert bei einer Abtastrate von $25 \mathrm{MHz}$ und 8192 Abtastpunkten etwa $8 \mathrm{~s}$.

\section{Simulation und Rekonstruktions- Algorithmus}

In der entwickelten Simulationsumgebung werden Bildmatrizen eingelesen, in denen die unterschiedliche Schallgeschwindigkeiten bzw. Dämpfungen durch unterschiedliche Pixelwerte dargestellt werden. Für die Berechnung der Projektionen aus verschiedenen Blickwinkeln wird eine Fächerstrahl-Geometrie mit einstellbarem Öffnungswinkel angenommen. Entlang der Pixelstrecken zwischen Sender und Empfänger werden jeweils die Pixelwerte aufsummiert. Die Anzahl der verwendeten Wandler kann dabei variiert und zusätzlich kann zwischen den einzelnen Wandlern interpoliert werden, um Daten für die Bereiche zwischen den Wandlern zu generieren. Um aus den einzelnen Projektionen ein Schnittbild zu erzeugen, wird ein klassischer Rückprojektionsalgorithmus nach Radon [16] verwendet. Zur Nutzung der in Matlab bereits vorhandenen zeitoptimierten Funktion zur RückprojektionsRekonstruktion (inverse Radontransformation) muss die Fächerstrahl- in eine Parallelstrahlgeometrie umgerechnet werden (Rebinning). Hierdurch erhält man eine Darstellung aller Projektionen über einen Blickwinkel (Sinogramm). Anschließend wird daraus ein 2DSchnittbild rekonstruiert und ausgegeben.

Durch die Simulation ist es einerseits möglich, reale Ergebnisse abzuschätzen, und auf der anderen Seite kann der Simulationsalgorithmus anhand echter Messdaten verbessert werden. Somit kann bereits vorab die Tauglichkeit des verwendeten US-PT-Systems für bestimmte Gegebenheiten (Wandleranzahl, Öffnungswinkel) und Materialsysteme aber auch die Verwendung verschiedener Korrekturfunktionen, wie Interpolation oder Filterung des Sinogramms im Fourierraum (gefilterte Rückprojektion), überprüft werden.

\section{Kalibrierung}

Da die Vorlaufstrecken für die im entwickelten System verwendeten US-Wandler manuell gefertigt wurden, kommt es dabei teilweise zu geometrischen Unterschieden. So wirken sich die Länge der Vorlaufstrecke und inre Temperaturabhängigkeit auf die gemessene Schallgeschwindigkeit aus. Je nach Verklebung der Piezo-Kristalle an den Vorlaufstrecken können die US-Wandler zudem unterschiedliche Sende- und Empfangscharakteristiken aufweisen. Um diese Faktoren zu berücksichtigen, muss das System entsprechend kalibriert werden. Dadurch ist die Angabe von absoluten Werten für Schallgeschwindigkeit und Dämpfung möglich. Für die Kalibrierung der Dämpfung wird ein Rundstrahlwandler in der Mitte des Tomografen verwendet. Die einzelnen US-Wandler 
erhalten Korrekturfaktoren, welche sie untereinander vergleichbar machen.

Als Kalibrationsmedium eignet sich destilliertes Wasser, da für dieses sowohl Schallgeschwindigkeit als auch Dämpfung bei definierten Temperaturen bekannt sind.

\section{Messablauf}

Der Ablauf der Messungen mit realen Medien erfolgt analog zur Simulation, weshalb der gleiche Algorithmus mit einigen Erweiterungen zum Einsatz kommt. Um im US-PT-System die benötigten Projektionen aus verschiedenen Blickwinkeln zu erzielen, senden alle Wandler nacheinander ein Signal aus, während sich die anderen 39 Wandler im Empfangsmodus befinden. Der Projektionswinkel wird also im Gegensatz zur klassischen CT nicht mechanisch, sondern elektronisch variiert. Sollte die Dämpfung des verwendeten Mediums, wie etwa der Kunststoffschmelze zu groß sein, können bis zu vier Wandler gleichzeitig als Sender betrieben werden. Ebenso ist ein Schwenk des Schallfelds möglich, indem mehrere Wandler phasenverschoben angesteuert werden.

Da Brechung und Beugung in erster Näherung in den betrachteten Kunststoffschmelzen vernachlässigbar sind, wird hier ebenfalls angenommen, dass sich der Transmissionspuls weitgehend geradlinig zwischen den Sender/Empfänger-Paaren ausbreitet. Die Streuung muss bei gefüllten Schmelzen ebenfalls berücksichtigt werden. Die gestreuten USSignale werden aber in der Regel später als der Transmissionpuls detektiert.

Durch Einstellung des Öffnungswinkels wird bestimmt, von welchen Sender/EmpfängerPaaren die aufgenommenen US-Signale für die Schnittbilderzeugung ausgewertet werden. Die als Amplituden-Scans (A-Scans, vgl. Abb. 3) aufgenommenen Signale werden mit einem Bandpass (Finite Impulse Response) mit schmaler Bandbreite um die Sendefrequenz bzw. der dritten Harmonischen gefiltert, um störende Rauschsignale zu unterdrücken. Anschließend wird für jeden Signalpuls mit Hilfe eines speziellen Algorithmus auf Basis der Schwellwertdetektion eine Laufzeit errechnet. Diese gibt an, wann der US-Puls den Empfänger erreicht hat. Der Schwellwert muss dabei je nach Medium und Signal-zu-RauschVerhältnis (SNR) angepasst werden. Letzteres kann entsprechend durch wiederholte Messungen verbessert werden. Anhand der Laufzeit und des bekannten Abstands des Sender/Empfänger-Paares wird eine Schallgeschwindigkeit berechnet.

Die Intensität der Pulszüge wird zur Ermittlung der Dämpfung verwendet. Hierzu wird allerdings ein realer Referenzpulszug benötigt.
Dies kann entweder - sofern messbar - ein Rückwandecho (siehe Abb. 3) oder ein Transmissionspuls aus einer Referenzmessung sein. Zusätzlich kann optional zwischen den Messwerten nebeneinander liegender Empfänger interpoliert werden, um auch für Bereiche ohne US-Wandler oder auch für defekte Wandler Messwerte zu erzeugen. Anschließend wird von der Software ein Schnittbild rekonstruiert, welches die Verteilung der Schallgeschwindigkeit bzw. der Dämpfung im Tomografen anzeigt.

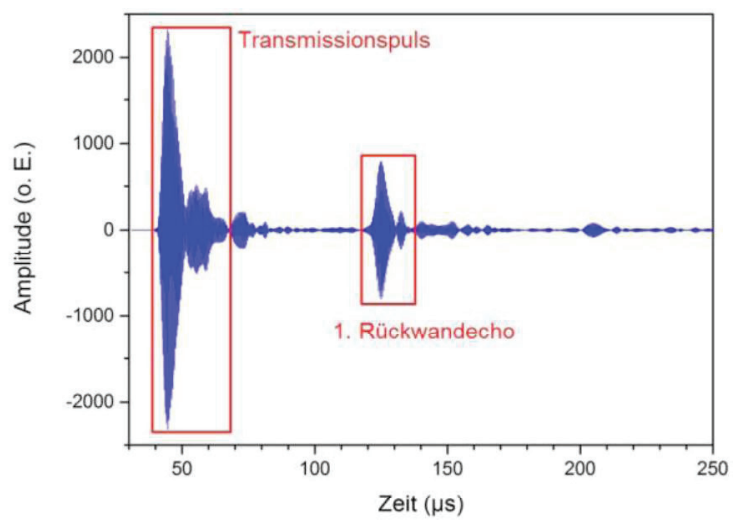

Abb. 3: Bandpass-gefilterter A-Scan eines US-Signals zweier gegenüberliegender Wandler im mit destilliertem Wasser gefüllten US-PT-Adapter: Der Transmissionspuls und das erste Rückwandecho sind rot markiert.

\section{Ergebnisse}

\section{Simulationen von Agglomeraten und Gradienten}

Die Auswirkung unterschiedlicher Öffnungswinkel der Wandler als auch die Verwendung der Interpolation auf die Rekonstruktion wird anhand von Simulationsbildern verschiedener Problemstellungen in Abb. 4 (Agglomerate) und Abb. 5 (verschobener Gradient) exemplarisch demonstriert. Dabei ist ebenfalls zu erkennen, dass der beim Aufbau des Messsystems eingegangene Kompromiss aus Anzahl und Apertur der Wandler zu tomografischen Artefakten, wie z. B. Ringartefakten, Schattenwürfen oder Unschärfen, führt. Diese müssen durch geeignete Filterung oder Korrekturen beseitigt werden. Die Auflösung der Bildgebung ist durch die $2 \mathrm{~mm}$ breiten US-Wandler limitiert, so dass Strukturen, welche näher zusammen liegen, nicht mehr sauber getrennt werden. Ebenso ist zu erkennen, dass erst mit zunehmendem Öffnungswinkel auch der Außenbereich des Tomografen besser rekonstruiert werden kann. Bei realen Messungen muss daher geprüft werden, welche Signalabschwächung im entsprechenden Medium bei Öff- 
nungswinkeln größer $30^{\circ}$ noch akzeptabel ist bzw. wo noch Pulse detektiert werden können, um eine bessere Rekonstruktion zu ermöglichen.
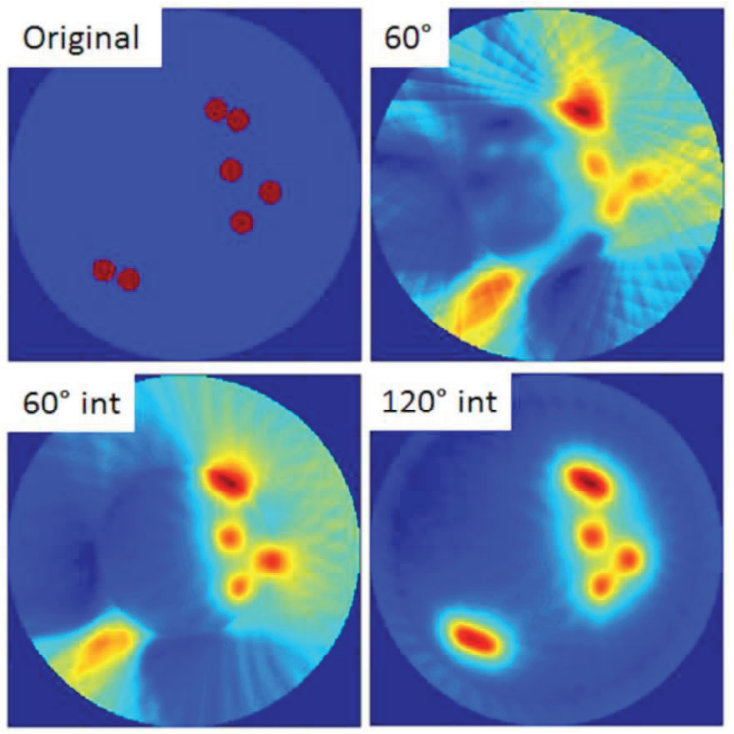

Abb. 4: Simulation einer Rekonstruktion von räumlich verteilten Agglomeraten: Bei den Rekonstruktionsbildern ist angegeben, welcher Öffnungswinkel verwendet und ob interpoliert (int) wurde.

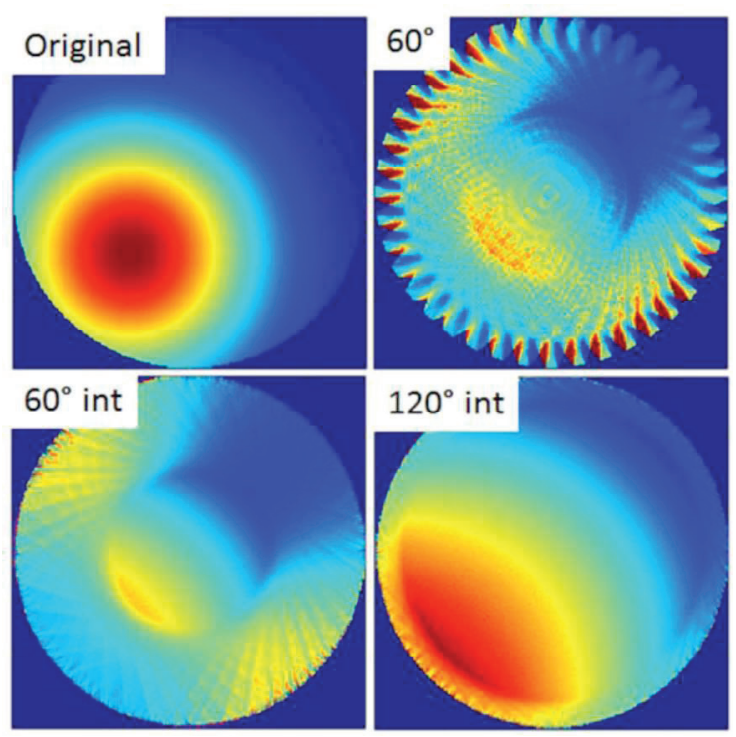

Abb. 5: Simulation einer Rekonstruktion eines verschobenen Gradienten: Bei den Rekonstruktionsbildern ist angegeben, welcher Öffnungswinkel verwendet und ob interpoliert (int) wurde.

\section{Messungen an gefüllten PP-Schmelzen}

Bei tomografischen Messungen im Extrusionsprozess ist zu beachten, dass die Kunststoffschmelze während der Aufnahme der Messungen weiterfließt. Bei einer Aufnahmedauer von $8 \mathrm{~s}$ beträgt die Strecke etwa $1 \mathrm{~cm}$, also $0,25 \mathrm{~mm}$ pro aktivem Sender (alle 0,2 s). Die Homogenität in Fließrichtung ist dabei essentiell, um Bewegungsartefakte bei der Rekonstruktion zu vermeiden. Versuche an PPSchmelze wiesen sowohl eine stärkere Signaldämpfung als auch mehr Rauschen als Vorversuche mit Wasser auf. Entsprechend gestaltete sich hier eine Signaldetektion deutlich schwieriger. Das Durchschallen von $60 \mathrm{~mm}$ gefüllter PP-Schmelze ist möglich und ein Transmissionspuls deutlich zu erkennen, jedoch ist dessen Länge nicht mehr einfach zu definieren. Ebenso kann das erste Rückwandecho nicht mehr detektiert werden. In Abb. 6 ist beispielhaft der A-Scan zweier gegenüberliegender Wandler bei der Durchschallung von mit $\mathrm{TiO}_{2}$ und Ruß gefüllter PP-Schmelze dargestellt. Die Festlegung der Länge des Transmissionspulses erfolgte hier anhand der erwarteten Länge aufgrund der Anzahl der verwendeten Burst-Wiederholungen.

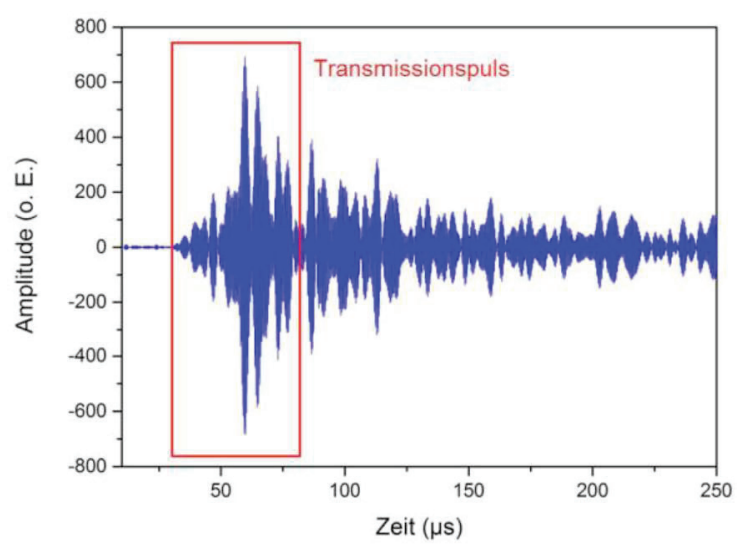

Abb. 6: Bandpass-gefilterter A-Scan eines US-Signals zweier gegenüberliegender Wandler im US-PT-Adapter: Das Medium war gefüllte PP-Schmelze. Der Transmissionspuls ist rot markiert.

Zudem konnten maximal Signale entsprechend einem Öffnungswinkel von $60^{\circ}$ ausgewertet werden, was eine niedrigere Bildqualität der Außenbereiche zur Folge hatte. Für die ermittelten Schnittbilder wurden jeweils Probekörper entnommen, welche als Vergleich dienten. Einige dieser Vollstäbe sind in Abb. 7 dargestellt. 


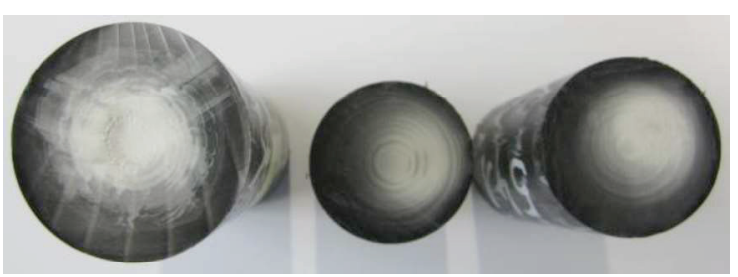

Abb. 7: Gradiert gefüllte Vollstäbe $\left(\mathrm{PP}+\mathrm{TiO}_{2}\right.$ + Ruß), welche bereits in Schmelzeform tomografisch untersucht wurden

Abb. 8 zeigt beispielhaft die Rekonstruktion der Schallgeschwindigkeit von reiner PP-Schmelze sowie gefüllter Schmelze mit eingebrachter Füllstoff-Gradierung. Dabei ist zu erkennen, dass der Bereich außerhalb des Öffnungswinkels stark artefaktbehaftet ist, v. a. nach Einbringen von Füllstoffen. Ebenso zeigt sich auch ein Einfluss der Füllstoffe auf die Schallgeschwindigkeit.
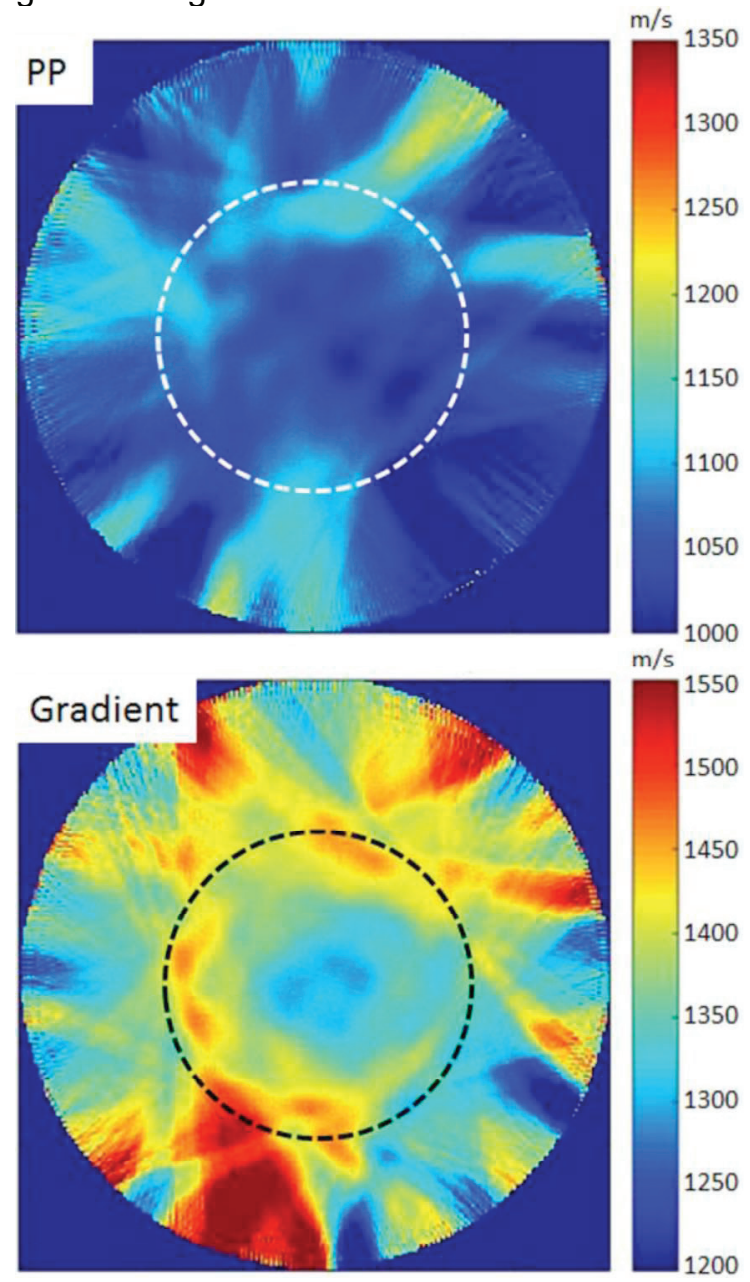

Abb. 8: Rekonstruktion der Schallgeschwindigkeit reiner (oben) sowie gefüllter PP-Schmelze mit Füllstoff-Gradierung. Aufgrund des Öffnungswinkels von $60^{\circ}$ ist nur der innere Bereich des Tomografen vollständig rekonstruiert (gestrichelte Markierung).
Im Allgemeinen lässt sich der Gradient (noch) nicht so fein bestimmen, wie er tatsächlich vorliegt. Weiterhin kann eine inhomogene Temperaturverteilung ebenfalls nicht ausgeschlossen werden, wenngleich die Untersuchung von reiner PP-Schmelze eine gewisse Homogenität zeigt. Die Rekonstruktion der Dämpfung gestaltet sich im Falle von PP ebenfalls schwieriger, da die jeweiligen Pulszüge nicht eindeutig zu definieren sind und Streueffekte ebenfalls einen stärkeren Einfluss haben (vgl. Abb. 6).

\section{Diskussion}

Die Untersuchung der lokalen Füllstoffverteilung war mit dem entwickelten System möglich. Dabei erforderte die Geometrie der hier untersuchten gradierten Vollstäbe einen Kompromiss aus Wandlergröße, -anzahl, Öffnungswinkel sowie möglicher Sendeleistung. Als Folge dessen liefert der vorliegende Tomograf aufgrund seines Öffnungnswinkels von bestenfalls $60^{\circ}$ lediglich in der Mitte eine ausreichende Bildqualität bei der Rekonstruktion. Hinzu kommt noch das Bestreben, eine Echtzeitkontrolle durchzuführen, wodurch ein weiterer Kompromiss zwischen Geschwindigkeit und Rekonstruktionsqualität notwendig ist. Die Genauigkeit der Laufzeitdetektion mit dem aktuellen Algorithmus beträgt $0,1-1 \mu \mathrm{s}$ je nach Materialsystem und Füllstoffanteil. Die Beschränkung der Signale auf definierte Zeitfenster sorgt dabei für einen Zeitgewinn. Dies kann durch Optimierung von Abtastfrequenz und Anzahl der Abtastpunkte bei der Signalaufnahme erreicht werden. Allerdings geht dies auf Kosten der Zeitauflösung und die ungefähren Signallaufzeiten müssen bekannt sein.

Mit dem verwendeten Aufbau konnte gefüllte PP-Schmelze trotz $60 \mathrm{~mm}$ Schmelzekanaldurchmesser mit nur einem US-Wandler durchschallt werden. Allerdings ist hierfür aufgrund der stärkeren Signaldämpfung eine größere Signalverstärkung notwendig, wodurch auch das Rauschen verstärkt wird. Somit ist wiederum eine höhere Anzahl an Messwiederholungen notwendig, um ein ausreichendes SNR zu erhalten.

Die durch den nicht optimalen Öffnungswinkel erzeugten Artefakte (Ringe, Schatten...) können durch Filterung und Interpolation nur teilweise behoben werden. Einen weiteren Ansatz bietet hier ein iteratives Rekonstruktionsverfahren, welches allerdings mehr Rechenleistung benötigt. Dies wäre allerdings unter parallelisierter Nutzung von hierfür optimierten Mehrkern-Prozessoren möglich. Dadurch kann die gesamte Messdauer auch weiter verkürzt werden. 


\section{Zusammenfassung}

Das entwickelte US-PT-System hat die grundsätzliche Möglichkeit aufgezeigt, die lokale Verteilung von Füllstoffen in Kunststoffschmelze ortsaufgelöst zu detektieren. Werden sämtliche Einflüsse beim Rekonstruktionsalgorithmus einbezogen oder korrigiert, ist es bei entsprechender Kalibrierung des Systems möglich, neben der Füllstoffverteilung auch die lokale Temperaturverteilung darzustellen. Durch Zusammenfügen der einzelnen Schnittbilder kann anschließend ein 3D-Bild entlang des Vollstabes erzeugt werden. Die USTomografie besitzt somit großes Potential für weitere Anwendungen zur Inline-Prozessüberwachung von Kunststoffschmelzen.

\section{Danksagung}

Das Forschungsvorhaben VIII/3-3852/45/5 wurde durch das Bayerische Staatsministerium für Wirtschaft, Infrastruktur, Verkehr und Technologie (BStMWIVT) gefördert. Wir bedanken uns für die finanzielle Unterstützung.

\section{Literaturnachweis}

[1] T. Hochrein, I. Alig, Prozessmesstechnik in der Kunststoffaufbereitung, Vogel Buchverlag (2011)

[2] V. Deutsch, M. Platte, M. Vogt, Ultraschallprüfung: Grundlagen und Industrielle Anwendungen, Springer Verlag, Berlin (1997)

[3] V. Deutsch, Die Ultraschallprüfung, 2. Auflage, Castell-Verlag (2012)

[4] F. Dinger, Ultraschallverfahren für Prozesskontrolle und Qualitätsüberwachung, Plastverarbeiter 11, $92-94$ (2007)

[5] I. Alig, D. Lellinger, R. Lamour, J. Ramthun, Inline-Monitoring mit Ultraschall, Kunststoffe 90, $96-100$ (2000)

[6] I. Alig, In-Line Prozessmonitoring am Zweischneckenextruder mittels Ultraschall, DKI, Veröffentlichung für das Fraunhofer LBF, Land Bayern, Externe Projektnummer: Bayern 917 (2001)

[7] I. Alig, D. Lellinger, B. Steinhof, D. Fischer, Ultraschallspektroskopie zur Inline-Kontrolle, Kunststoffe 5, 60 - 65 (2006)

[8] Y-B. Zhu, N-Y. Ning, Y. Sun, Q. Zhang, Q. Fu, A new technique for preparing a filled type of polymeric gradient material, Macromolecular Materials and Engineering 11/291, 1388 - 1396 (2006); doi: 10.1002/mame.200600249

[9] G. Wu, B. Wen, S. Hou, Preparation and structural study of polypropylene-talc gradient materials, Polymer International 53, 749 - 755 (2004); doi: 10.1002/pi.1360

[10] C-K. Sun, L-J. Chen, H-W. Chen, Plastic waveguide for terahertz wave, United States Patent US 7409132 B2 (2008)
[11] M. Krumova, C. Klingshirn, F. Haupert, K. Friedrich, Microhardness studies on functionally graded polymer composites, Composites science and technology. 61, $557-563$ (2001); doi: 10.1016/S0266-3538(00)00228-1

[12] C. Hirt, M. Lang, K. Kretschmer, P. Heidemeyer, M. Bastian, Grashalm als Vorbild - Gradientenwerkstoffe mit gezieltem Verlauf der Eigenschaftskennwerte, Kunststoffe 3, 109 - 112 (2015)

[13] G. Schwarzenberg, Untersuchung der Abbildungseigenschaften eines $3 D$-UltraschallComputertomographen, Dissertation (2008); doi: 10.5445/KSP/1000011990

[14] B. Praher, K. Straka, G. Steinbichler, Schmelzetemperatur mit Ultraschall messen, Kunststoffe 7, $45-49$ (2013)

[15] H. C. Schröder, Prüfen von Altanlagen und Komponenten auf kritische Fehler - Chancen der ZfP, 30. FDBR Fachtagung „Rohrleitungstechnik", Magdeburg (2015)

[16] A. Kak, M. Slaney, Principles of Computerized Tomographic Imaging, Society of Industrial and Applied Mathematics (2001); doi: $10.1137 / 1.9780898719277$ 